The term 'manipulation' may refer to a therapeutic manoeuvre "used to designate instances of crude efforts to run the patient's life" (Stewart, 1985). This practice has only qualified support. Aside from describing what therapists do, the term is more often used to describe coercive behaviour by patients. Frequently descriptions of 'manipulative patients' are overtly slanderous. 'Manipulation', when loosely used, tends to imply: (a) the patient has engaged in a negative behaviour ('manipulative' implying he/she does so habitually) that angers the therapist; (b) he/she has done so purposefully (as opposed to responsively); (c) the behaviour should be resisted or countered by staff - supposedly for the benefit of the patient (this is often done by staff attempting to out-manoeuvre or out-manipulate the patient).

This process goes against the very essence of suicide prevention as it implies therapeutic defiance rather than a therapeutic alliance. This will be fuelled by poorly contained countertransference hate. The jargon of the concept fuels the rationalisation - giving a misguided sense of understanding.

I propose that the term 'manipulation' be dropped, as all too often it is used in a way that is not only unhelpful, but hazardous. The degree of understanding of patient behaviour fostered by this term is that which might be expected of lay people. Professionals would do better recognising that acting out by distressed patients is usually largely responsive rather than solely purposeful. Behavioural concepts of reinforcement and dynamic concepts of defence mechanisms provide much deeper levels of understanding. They also permit the fostering of therapeutic alliances rather than confrontations when staff endeavour to help difficult, distressed patients.

Stewart, R. (1985) Psychoanalysis and psychoanalytic psychotherapy. In Comprehensive Textbook of Psychiatry (eds H.I. Kaplan \& B.J. Sadock). Baltimore: Williams and Wilkins.

Chris TAYLOR

Suicide Research and Prevention Programme

Princess Alexandra Hospital

Brisbane Qld 4102

Australia

\section{Treating negative symptoms}

SIR: The treatment of negative symptoms in schizophrenic patients is a difficult clinical problem; thus, positive clinical trials in this area, such as that of Duinkerke et al (BJP, October 1993, 163, 451-455), are always of interest. However, some methodological questions arise from this study.
The use of type II schizophrenia as an inclusion criterion is unclear. Crow (1981) has pointed out that he regards type I and type II as symptom clusters rather than patient types. Thus it is uncertain whether the authors operationalised the concept of type II schizophrenia and also uncertain whether the presence of type I (positive) symptoms constituted an exclusion criterion. In this respect, and also to estimate the generalisability of the study, it would be interesting to know what proportion of the patients screened met the study entry criteria. The use of a Wilcoxon signed rank test to examine the difference between a 'positive' and 'negative' BPRS subscore is problematic unless clinical significance has somehow been assigned $a$ priori to such a difference.

Further questions arise concerning the statistical procedures. The number of patients studied was small (33) and no confidence intervals or power calculations are provided. There are also no data on the inter-rater reliability of the SANS in this study, which apparently took place in six centres. No correction has been made for multiple significance testing of the individual SANS items; however, since these items are not statistically independent, a simple Bonferroni correction would be too conservative. Resolution of this problem depends in part on which variables were defined as primary before the study was performed (Oakes, 1993).

Accepting nonetheless that a true effect of ritanserin has been shown, this could be due to an effect on at least two other factors different from but often confused with negative symptoms: extrapyramidal symptoms (EPS) and depression. The authors themselves quote evidence that ritanserin acts on both. In this study, a depression rating scale was not used, but there was a significant effect on the BPRS item 'depressed mood'. As for EPS, low scores on the Simpson-Angus scale do not exclude neuroleptic-induced phenomena such as poverty of gesture or mask-like facial expression, since these do not appear in the scale. None of the patients in the study was taking anti-Parkinsonian medication, so the presence of EPS must be seriously considered. In this respect it would be interesting to know the average daily neuroleptic dose in chlorpromazine equivalents.

Finally, although such an 'add-on' study neatly avoids the ethical difficulties of placebo treatment, it raises the problem of pharmacokinetic interactions. Serum neuroleptic levels were not measured and therefore a pharmacokinetic effect cannot be ruled out. The use of ten different neuroleptics (of five different chemical classes) in 33 patients is a further complication. 
Crow, T.J. (1981) Positive and negative schizophrenia symptoms and the role of dopamine. British Journal of Psychiatry. 139, 251-254.

OAKFs, M. (1993) The logic and role of meta-analysis in clinical research. Statistical Methods in Medical Research, 2, 147-160.

Paul Bailey

FABRICE DUVAL

Centre Hospitalier

F-68250 Rouffach

France

AUTHORS' REPLY: Apparently, some aspects of our study need clarification.

Each participating psychiatrist selected some outpatients with schizophrenia who were receiving stable neuroleptic medications, and who had predominantly negative symptoms. Thus selection was based on symptom severity; whether this reflects different syndromes of schizophrenia is still a matter of discussion, in which we do not want to take a position now. After selection, patients could decide to participate, and the positive and negative symptoms were rated with the SANS and BPRS. The average ratings showed that this selection procedure was satisfactory.

The single primary parameter of this trial was change in the total SANS score at endpoint. In the ritanserin group, a larger reduction was found in the total SANS score than in the placebo group (at endpoint $P=0.012$ ): the $95 \%$ confidence intervals were -15.0 to -5.5 in the ritanserin group, and -7.6 to 2.5 in the placebo group.

In our paper we cited a study in which no influence of ritanserin on liver enzymes was found. Therefore pharmacokinetic interactions between ritanserin and other neuroleptics are not likely, but cannot be ruled out. The measurement of plasma concentrations of the neuroleptics and ritanserin is necessary in future investigations.

In this trial, ritanserin reduced the SANS scores (i.e. reduced negative symptoms). Could this be explained by antidepressant or anti-Parkinsonian actions? Does ritanserin reduce all negative symptoms, or only some? Answering these questions was not the primary aim of our trial, and a trial with only 33 patients cannot give conclusive evidence. But the results obtained give indications for possible answers. We do not think that the ritanserin-induced effect of this study is mainly an anti-Parkinsonian effect, since the patients had a low EPS (Simpson-Angus) scores at baseline, and the small, random changes in this score was not correlated to the larger changes in the SANS score. An antidepressant effect of ritanserin cannot be ruled out.
There is no a priori reason that a drug with a new mode of action (such as ritanserin) fits into existing clinical categories. The only way to evaluate is to rate its effects on a large variety of symptoms. Having found an effect on the primary parameter (total SANS), we wanted to present the changes in the various SANS items (including the statistical evaluation) - we did not use the testing of the individual items to construct a primary effect. The criticism of 'multiple testing' is therefore not appropriate. Some negative symptoms were greatly reduced in the ritanserin group, and others very little. If this finding can be replicated, the clinical actions of ritanserin are more precisely defined. The question then raised is whether negative symptoms form a unitary syndrome.

To evaluate the clinical effects of ritanserin (or drugs with a similar mode of action), changes in a variety of symptoms in a large group of patients must be tested. The number of patients in our study was too small for such conclusions, but the listed symptoms give an impression of which symptoms could be especially relevant in future larger studies.

$$
\begin{array}{r}
\text { S.J. DUINKERKE } \\
\text { P.A. BOTTER } \\
\text { A.A.I. JANSEN } \\
\text { P.A.M. VAN DONGEN } \\
\text { A.J. VAN HAAFTEN } \\
\text { A.J. BOOM } \\
\text { J.H.M. VAN LAARHOVEN } \\
\text { H.L.S.M. BUSARD }
\end{array}
$$

Janssen Pharmaceutica B. $V$.

Dr Paul Janssenweg 150

PO Box 90240

5000 LT Tilburg

The Netherlands

\section{Tardive dyskinesia as a risk factor for negative symptoms}

SIR: Liddle et al (BJP, December 1993, 163, 776780 ) conclude that there is an association between the negative symptoms of schizophrenia, orofacial tardive dyskinesia (TD), and increasing age. Their conclusions may be unwarranted, because the group of schizophrenic patients studied was atypical, and the definition of TD did not require antipsychotic treatment.

The subjects were atypical because they were long-stay patients (length of stay was not stated) and thus likely to have more severe schizophrenia and, in particular, to have prominent negative symptoms. The effects of institutionalisation are not 